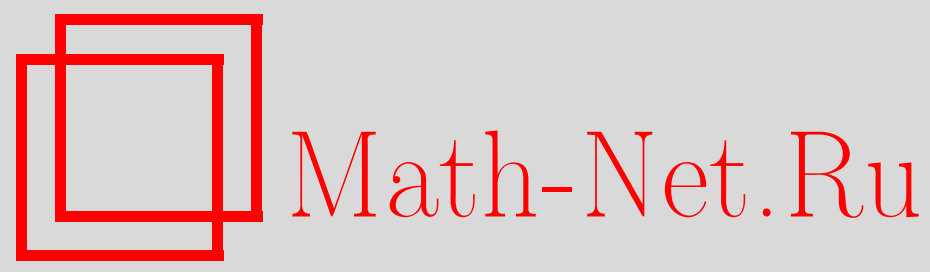

А. Р. Алимов, Выпуклость чебышёвских множеств, содержащихся в подпространстве, Матем. заметки, 2005, том 78 , выпуск 1, 3-15

DOI: https://doi.org/10.4213/mzm2555

Использование Общероссийского математического портала Math-Net.Ru подразумевает, что вы прочитали и согласны с пользовательским соглашением http://www.mathnet.ru/rus/agreement

Параметры загрузки:

IP : 3.85 .183 .62

26 апреля 2023 г., $11: 24: 17$

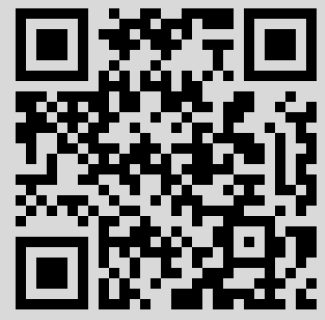




\section{ВЫПУКЛОСТЬ ЧЕБЫШЁВСКИХ МНОЖЕСТВ, СОДЕРЖАЩИХСЯ В ПОДПРОСТРАНСТВЕ}

\section{А.Р. Алимов}

Рассматривается задача о выпуклости чебышёвского множества $M$ в линейном нормированном или несимметрично нормированном пространстве $(X,\|\cdot\|)$ при дополнительном условии $M \subset H$, где $H$ - подпространство в $X$. Пусть $B$ - единичный шар в $X$. Устанавливается, что если $|\cdot|_{H, \theta}$ - несимметричная норма на $H$, определяемая функционалом Минковского множества $(B-\theta) \cap H$ относительно 0 , где $\|\theta\|<1$ произвольно, то $M$ - чебышёвское множество в $\left(H,|\cdot|_{H, \theta}\right)$ при любом выборе $\theta$. Исходя из этого утверждения даются достаточные признаки и необходимые признаки выпуклости чебышёвских множеств $M$ и ограниченных чебышёвских множеств $M$ в $X$ при условии $M \subset H$.

Библиография: 21 название.

1. Введение. Подмножество $M$ пространства $(X,\|\cdot\|)$ назьвается чебышёвским множеством, если для каждой точки $x \in X$ множество

$$
P_{M} x=\{y \in M \mid\|x-y\|=\rho(x, M)\}
$$

ее ближайших точек из $M$ состоит из одной точки. Здесь $\rho(x, M)=\inf _{z \in M}\|x-z\|-$ расстояние от $x$ до $M,\|\cdot\|$ - норма или несимметричная норма на линейном пространстве $X$. О чебьшёвских множествах см. [1]-[6].

Известно, что решение задачи о выпуклости чебьшёвских множеств зависит от структурных ограничений, накладьваемых на рассматриваемые множества. К примеру, И.Г. Царьков [4], [5] показал, что класс конечномерных линейных нормированных пространств, в которых всякое чебьшёвское множество выпукло, отличен от класса пространств, где всякое ограниченное чебышёвское множество вьпукло. У нас подобньп ограничением будет условие, что чебьшёвское множество $M$ содержится в некотором фиксированном подпространстве $H$ пространства $X$. Подчеркнем, что наша задача отличается от классической проблемы приближения подпространствами.

Задача приближения множествами $M$ с ограничением $M \subset H$, где $H$ - фиксированное подпространство, является новой. Такую постановку задачи недавно рассматривал А. Л. Браун $[7, \S 4]$, установивший $P$-ацикличность солнц $M$ в конечномерном линейном нормированном пространстве $X$ при условии $M \subset H, H \subset X$ - двумерное подпространство (см. следствие 1 ниже).

Работа выполнена при поддержке Российского фонда фундаментальных исследований, грант № 05-01-00055. 
В работе всегда будет предполагаться, что $X=(X,\|\cdot\|)$ - действительное линейное несимметрично нормированное пространство (определение см. ниже; в частности, в качестве $X$ можно рассматривать линейное нормированное пространство), $\operatorname{dim} X \leqslant \infty$ (случай $\operatorname{dim} X<\infty$ всегда будет оговариваться отдельно), $H$ - собственное аффинное подпространство в $X$.

Сформулируем основные результаты работы. В теоремах 1 и 2 формулируются достаточные условия на пространство $X$, при которых всякое чебьшёвское множество $M$ в $X$, содержащееся в заданном подпространстве $H \subset X, \operatorname{dim} H<\infty$, выпукло.

Ниже $B$ - единичньй шар пространства $X$. Как обычно, для вьпуклого тела $C \subset X$ посредством sm $C$ мы обозначаем множество его точек гладкости, а посредством $\exp C-$ множество его достижимых точек (подробнее см. раздел 2); bd $C$ - его граница.

Пусть $H$ - подпространство в $X$. Положим $|\cdot|_{H, \theta}-$ несимметричная норма на $H$, определяемая на $H$ функционалом Минковского тела $(B-\theta) \cap H,\|\theta\|<1$. Определим $S_{H, \theta}=\left\{\left.z \in H|| z\right|_{H, \theta}=1\right\}=\operatorname{bd}_{H} B_{H, \theta}-$ единичная сфера в $\left(H,|\cdot|_{H, \theta}\right)$.

Teоpema 1. Пусть $H \subset X-$ nодпространство, $\operatorname{dim} H<\infty$, и пусть $M \subset H-$ чебышёвское мнохество в $X$. Предположим, что найдется такая точка $\theta \in X$, $\|\theta\|<1$, что выполнено хотя бы одно из следующих условий:

a) $\operatorname{sm} B_{H, \theta}=B_{H, \theta}$ (m.е. любая точка сферы $S_{H, \theta}$ является ее точкой гладкостu);

б) если $\operatorname{dim} H=3$ или 4, то $\exp B_{H, \theta} \subset \operatorname{sm} B_{H, \theta}$ (m.e. любая достижимая точка шара $B_{H, \theta}$ является его точкой гладкости).

Тогда множество $М$ выпукло.

Пусть $\operatorname{dim} H=2$. Рассмотрим следующую несимметричную норму $|\cdot|_{H, \theta}$ на $H$, порожденную функционалом Минковского тела $(B-\theta) \cap H,\|\theta\|<1$. Положим $\mathfrak{X}=\{(H$, | $\left.\left.\left.\cdot\right|_{H, \theta}\right)\right\}_{\|\theta\|<1}-$ пространство $H$ с заданной на нем системой несимметричных норм $|\cdot|_{\text {н, }}$. Множество $M \subset H$ назьвается чебышёвским относительно $\mathfrak{X}$, если $M$ является чебышёвским множеством в пространстве $\left(H,|\cdot|_{H, \theta}\right)$ для любого $\theta$. Если $\mathfrak{X}$ состоит только из одного пространства, то это определение совпадает с обычным определением чебьшёвского множества. Пусть $S_{H, \theta}=\left\{\left.z \in H|| z\right|_{H, \theta}=1\right\}$ - единичная сфера в $H$ относительно $|\cdot|_{H, \theta}$. Система точек $\mathscr{P}:=\left\{p_{\theta} \in S_{H, \theta}\right\}_{\|\theta\|<1}$ называется $\partial о$ стижимой относительно $\mathfrak{X}$, если для тела $W_{\mathfrak{X}}:=\overline{\operatorname{conv}}\left(\bigcup_{\|\theta\|<1}\left\{S_{H, \theta}-p_{\theta}\right\}\right)$ точка 0 принадлежит гранище тела $W_{\mathfrak{X}}$ и является достижимой; система точек $\mathscr{P}$ назьвается гладкой, если точка $0 \in \mathrm{bd} W_{\mathfrak{X}}$ является точкой гладкости тела $W_{\mathfrak{X}}$. Здесь $\overline{\mathrm{conv}} W-$ замькание вьпуклой оболочки $W$.

В ослабленном виде сформулируем теорему 2.

Пусть $H \subset X-$ подпространство, $\operatorname{dim} H=2$. Тогда если любое чебышёвское множество $M \subset H$ в $X$ выпукло, то любая достижимая относительно набора $\mathfrak{X}=\left\{\left(H,\|\cdot\|_{H, \theta}\right)\right\}_{\|\theta\|<1}$ система точек $\left\{p_{\theta} \in S_{H, \theta}\right\}_{\|\theta\|<1}$ является гладкой.

В теореме 3 мы даем достаточное условие на пространство $X$, при котором всякое ограниченное чебышёвское множество $M \subset H$ в $X$ выпукло. Сформулируем ее также в ослабленном виде.

Пусть $\operatorname{dim} X<\infty, H$ - аиперплоскость в $X$ и пусть $M \subset H$ - ограниченное чебышёвское множество в $X$. Если хотя бъ для одного из функиионалов $h_{1}, h_{2} \in S^{*}$, 
определяюших $H$, выполнено $h_{i} \in \operatorname{int}_{S^{*}} \overline{\exp }\left(S^{*}\right), i=1$ или 2, то множество $M$ выпукло. Здесь $S^{*}$ - единичная сфера пространства, сопряженного к $X$.

Утверждение теоремы 4 берет начало от результата Царькова [5], построившего норму на линейном пространстве $X, 3 \leqslant \operatorname{dim} X<\infty$, относительно которой всякое ограниченное чебышёвское множество в $X$ выпукло, в то время как существует невыпуклое неограниченное чебышёвское множество в $X$.

Teорема 4. Пусть $\operatorname{dim} X<\infty, H \subset X-$ гиперплоскость в $X, \operatorname{dim} H \geqslant 3$. Тогда на $X$ существует норма, относительно которой любое ограниченное чебышёвское мнохество $M \subset H$ в $X$ будет выпукло, однако найдется неограниченное невыпуклое чебышёвское мнохсество $M_{1} \subset H$ в $X$.

\section{2. Определения и вспомогательные утверждения.}

Линейные пространства $c$ несимметричной нормой. Пусть $X$ - действительное линейное пространство. Несимметричное расстояние $\rho: X \times X \rightarrow \mathbb{R}_{+}$на $X$ определяется посредством функционала Минковского некоторого фиксированного вьпуклого ограниченного тела $B$ - единичного шара - содержащего начало координат в своей алгебраической внутренности. Именно, для $x, y \in X, x \neq 0$,

$$
\rho(0, x):=\sup \{\sigma \geqslant 0 \mid x \notin \sigma B\}, \quad \rho(x, y):=\rho(0, y-x), \quad \rho(0,0)=0 .
$$

Функция $\|x\|=\rho(0, x)$ назьвается несимметричной нормой, она удовлетворяет всем аксиомам нормы, исключая аксиому симметрии; в общем случае $\rho(x, y) \neq \rho(y, x)$, т.е. $\|y-x\| \neq\|x-y\|$. Неравенство треугольника выполнено в следующем виде:

$$
\rho(x, y) \leqslant \rho(x, z)+\rho(z, y)
$$

для любых $x, y, z \in X$. В случае, если функционал $\|\cdot\|$ симметричен, он является нормой.

Если $x \in X, r>0$, то определим

- $B(x, r)=\{y \in X \mid\|y-x\| \leqslant r\}$ - замкнутьй шар;

- $\stackrel{\circ}{B}(x, r)=\{y \in X \mid\|y-x\|<r\}$ - открытьй шар;

- $S(x, r)=\{y \in X \mid\|y-x\|=r\}-$ cфера

соответственно с центром $x$ и радиусом $r$. Единичный шар $B(0,1)$ обозначается посредством $B, \stackrel{\circ}{B}=\stackrel{\circ}{B}(0,1)$ - открытый единичньй шар, $S=S(0,1)$ - единичная сфеpa. Естественная $T_{1}$-топология на $X$ задается базой $\{\stackrel{\circ}{B}(x, r)\}, x \in X, r>0$; если $\operatorname{dim} X=n<\infty$, то эта топология совпадает с обычной евклидовой топологией на $\mathbb{R}^{n}$, однако в бесконечномерном случае она может быть даже неметризуемой.

Впервые вопросы геометрической теории приближений относительно несимметричных расстояний рассматривал, по-видимому, А. Брондстед [8], хотя в других задачах несимметричные расстояния возникали и ранее.

Сопряженное пространство к линейному несимметрично нормированному пространству. Пусть $(X,\|\cdot\|)$ - линейное пространство с несимметричной нормой $\|\cdot\|$. Линейный функционал $f$ на $X$ называется ограниченны.м, если величина

$$
\|f\|=\sup _{x \neq 0} \frac{f(x)}{\|x\|}
$$


- несимметричная норма функционала $f$ - конечна. Посредством $X^{*}$ обозначим множество всех линейных ограниченных функционалов на $X$. В общем случае $X^{*}$ не обязано быть линейным пространством, хотя всегда является выпуклым конусом (тем не менее, $X^{*}$ является линейньм пространством, если $\operatorname{dim} X<\infty$ или если несимметричная норма $\|\cdot\|$ на $X$ метризуема). Действительно, если $f, g \in X^{*}$, то $f+g \in X^{*}$ и $\lambda f \in X^{*}$ при всех $\lambda>0$, однако элемент $-f$ не обязан быть ограниченньм (и в [9, с. 331] это показано). Попутно заметим, что если $f \in X^{*}$, то $f(x) \leqslant\|f\| \cdot\|x\|$ для всех $x \in X$.

Как обычно, $B^{*}$ - единичный шар сопряженного пространства $X^{*}, S^{*}$ - единичная copepa.

Гладкие, достижимые и экстремальные точки. Несмотря на то, что мы рассматриваем несимметрично нормированные линейные пространства, многие факты из теории классических линейных нормированных пространств переносятся на несимметричньй случай без изменения. Напомним некоторые результаты из теории вьпуклых множеств.

Точка $x$, принадлежащая границе bd $C$ вьпуклого тела $C \subset X$, назьвается точкой гладкости $(x \in \operatorname{sm} C)$ тела $C$ (или его гранишы $\mathrm{bd} C)$, если опорная гиперплоскость к телу $C$ в точке $x$ единственна. Точка $x \in \mathrm{bd} C$ назьвается достижимой точкой тела $C$ (или его гранищы bd $C$ ), если существует гиперплоскость $H$ такая, что $H \cap C=\{x\}$; этот факт мы обозначаем $x \in \exp C$. Нульмерные грани тела $C$ назьваются его крайними (или әкстремальными) точками: $x \in \operatorname{ext} C$, если $x$ не принадлежит относительной внутренности никакого отрезка, лежащего в bd $C$.

Отметим, что если $x$ - точка гладкости шара $B$, то найдется (единственный) функционал $f \in S^{*}$ такой, что $f(x)=1$. В этом случае гиперплоскость $\left\{g \in X^{*} \mid g(x)=1\right\}$ является опорной гиперплоскостью к $f \in S^{*}$, которая пересекается со сферой $S^{*}$ в точности по элементу $f$. Поэтому $f \in \exp S^{*}$. Аналогично, если $x \in \exp S$ и $f \in S^{*}-$ функционал, которьй “достигает" $x$ (т.е. $\{z \in X \mid f(z)=1\} \cap S^{*}=\{x\}$ ), то $f \in \operatorname{sm} S^{*}$.

Нормы и несимметричные нормы в пространстве $X$ и на подпространстве $H$. Пусть $\theta \in \stackrel{\circ}{B}$. Введем следующие несимметричные нормы:

- $|\cdot| \theta$ - несимметричная норма на $X$, задаваемая функционалом Минковского тела $\stackrel{B}{B}-\theta$ относительно точки 0 ;

- $|\cdot|_{\text {, } \theta}-$ несимметричная норма на $H$, индуцированная несимметричной нормой $|\cdot| \theta \cdot$

Шар, сферу с центром $x$ и радиусом $r$ и т.п. объекты относительно несимметричной нормы $|\cdot|_{\theta}$ обозначим $B_{\theta}(x, r), S_{\theta}(x, r)$ и т.д.; подобным образом нижний индекс у некоторого объекта показьвает, относительно какой (несимметричной) нормы он определяется.

ПРЕДЛОЖЕНИЕ 1. Несимметричные нормы $\|\cdot\| u|\cdot|_{\theta}$ әквивалентны при любом выборе $\theta \in \stackrel{\circ}{B}$, причем

$$
\begin{aligned}
B_{\theta}(0, r) \subset B(0,1) & \Longleftrightarrow 0<r \leqslant(1+\|-\theta\|)^{-1}, \\
B(0, R) \subset B_{\theta}(0, r) & \Longleftrightarrow 0<R \leqslant r(1-\|\theta\|) .
\end{aligned}
$$

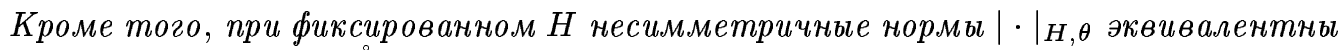
на $H$ для любого $\theta \in \stackrel{\circ}{B}$.

Мы докажем это утверждение чуть ниже. 
Обозначим через $\mathrm{H}_{x}^{k}(\cdot)$ гомотетию с центром $x \in X$ и коэффициентом $k \geqslant 0$.

Следующее важное в дальнейшем утверждение легко установить непосредственной проверкой.

ПРЕДЛОЖЕНИЕ А [4]. Пусть $\omega \in B(y, r), k \geqslant 0$. Тогда

$$
\mathrm{H}_{\omega}^{k}(B(y, r))=B(\omega+k(y-\omega), k r) .
$$

Для линейных нормированных пространств следующий факт широко известен (см., например, $[1$, предложение 0.2$])$. На случай несимметрично нормированньх пространств он переносится без изменений.

ПРЕДЛОЖЕНИЕ В. Пусть $x, x^{\prime} \in X, r, r^{\prime} \geqslant 0$. Тогда

$$
\stackrel{\circ}{B}(x, r) \subset \stackrel{\circ}{B}\left(x^{\prime}, r^{\prime}\right) \Longleftrightarrow\left\|x-x^{\prime}\right\| \leqslant r^{\prime}-r .
$$

ДоКАЗАТЕЛЬСТВо ПРЕДЛОЖЕНИЯ 1. По определению $B_{\theta}(0,1)=B(-\theta, 1)$. Далее, $B_{\theta}(0, r)=\mathrm{H}_{0}^{r}\left(B_{\theta}(0,1)\right)=\mathrm{H}_{0}^{r}(B(-\theta, 1))=B(-r \theta, r)$, где последнее равенство получено из (2). Применяя предложение В, получим $B_{\theta}(0, r)=B(-r \theta, r) \subset B(0,1)$ тогда и только тогда, когда $r \leqslant 1 /(1+\|-\theta\|)$, что доказьвает первое включение в $(1)$.

Второе включение в (1) доказьвается аналогично.

Солнца и строгие солнца. При изучении чебьшёвских множеств Н.В. Ефимов и С. Б. Стечкин [10] ввели важное понятие солнца, которое и само оказалось полезным в теории приближений: “солнечные” свойства множеств представляют самостоятельный интерес ввиду их связи с такими важными для теории приближений вопросами, как отделение выпуклых множеств, экстремальные точки, характеристика наилучшего приближения.

Напомним, что подмножество $M \subset X$ назьвается солнием, если для каждой точки $x \in X \backslash M$ существует точка $y \in P_{M} x$ такая, что

$$
y \in P_{M}[(1-\lambda) y+\lambda x] \quad \text { для всех } \lambda \geqslant 0,
$$

при этом точка $y$ назьвается солнечной точкой для $x$; множество $M$ назьвается $c m p o$ гим солнием, если для каждой точки $x \in X \backslash M$ множество $P_{M} x$ ее ближайших точек из $M$ непусто и утверждение (4) выполнено для каждой точки $y \in P_{M} x$. Ясно, что каждое строгое солнце является солнцем. Отметим, что в конечномерном пространстве каждое чебышёвское множество является солнцем [1, гл. 4].

Для $x, y, x \neq y$, опорньй конус к шару $B(x,\|y-x\|)$ в точке $y$ определяется как (см. [1, гл. 3]):

$$
\stackrel{\circ}{K}(y, x)=\bigcup_{\alpha>0} \stackrel{\circ}{B}(-\alpha y+(\alpha+1) x,(\alpha+1)\|y-x\|) .
$$

Важное свойство солнц - отделимость точки от солнца выпуклым конусом - дается в следующем утверждении (см., например, [1, гл. 3], [11], [8]).

Лемма А. Множество $M \subset X$ является солнием в $X$ тогда и только тогда, когда для каждой точки $x \notin M$ найдется точка $y \in P_{M} x$ такая, что $\stackrel{\circ}{K}(y, x) \cap$ $M=\varnothing$. 
3. Аппроксимативные свойства множеств, лежащих в подпространстве. Следующая лемма на первьй взгляд является неожиданной. Однако этот эффект обусловлен лишь тем, что мы не привыкли приближать относительно несимметричных расстояний, хотя они естественно возникают во многих задачах (см., например, [9], [12]-[15]). Наш вопрос о приближении множествами, лежащими в подпространстве, оказьвается одной из таких задач: сечение симметричного тела (шара $B$ ) аффинным подпространством $H$ вовсе не обязано быть симметричным! Это сечение определяет несимметричную норму на $H$, а множество $M \subset H$, если оно было чебышёвским множеством в $X$, по лемме 1 останется чебьшёвским множеством в $H$.

Лемма 1. Пусть $M \subset X$ является соответственно чебышёвским мно сеством, солнием или строгим солнием в пространстве $(X,\|\cdot\|)$. Тогда

1) множество $M$ обладает аналогичным свойством в пространстве $\left(X,|\cdot|_{\theta}\right)$ для любого $\theta \in \stackrel{\circ}{B}$;

2) если $\left(H,|\cdot|_{H, \theta}\right)$ - аффинное подпространство в $X$ и $M \subset H$, то $M$ обладает аналогичным свойством в пространстве $\left(H,|\cdot|_{H, \theta}\right)$ для любого $\theta \in \stackrel{\circ}{B}$.

Отметим без доказательства, что аналогичный результат верен также для $\alpha$-солнц и полусолнц (определения см. в [1], [2]).

В п. 2) леммы 1 существенным является то условие, что $M \subset H$. Легко построить примеры, показьвающие, что пересечение $M \cap H$, где $M$ - чебышёвское множество в $X$, $M \not \subset H$, не обязано быть чебьшёвским множеством в $H$.

ДокАЗАТЕЛЬСТво ЛЕМмы 1. 1) Пусть $M$ - чебышёвское множество в пространстве $(X,\|\cdot\|)$. Установим, что множество $M$ сохраняет это свойство в $\left(X,|\cdot|_{\theta}\right)$. Пусть $x \notin M$. По предложению 1 это влечет, что

$$
\rho_{\theta}(x, M):=\inf _{z \in M}|z-x|_{\theta}>0
$$

Без ограничения общности считаем $x=0, \rho_{\theta}(x, M)=1$.

Покажем, что $\rho(-\theta, M)=1$. По определению $B_{\theta}(0,1)=B(-\theta, 1)$. Поэтому $\stackrel{B}{B}(-\theta, 1)$ $\cap M=\varnothing$. Предположим противное: $\stackrel{\circ}{B}(-\theta, 1+\delta) \cap M=\varnothing$ для некоторого $\delta>0$. Из $(2)$ имеем, что $B(-\theta, 1+\delta)=B_{\theta}(\delta \theta, 1+\delta)$. Поскольку $|-\theta|_{\theta}<1-\varepsilon_{0}$ при некотором $\varepsilon_{0}>0$, то (3) нам дает, что $B_{\theta}(0,1+\varepsilon) \subset \stackrel{\circ}{B}(\delta \theta, 1+\delta)$ для всех $0<\varepsilon<\varepsilon_{0}$. Получили противоречие, поскольку, с одной стороны, $B_{\theta}(0,1+\varepsilon) \cap M=\varnothing$, а с другой стороны, $1=\rho_{\theta}(0, M)=\inf _{z \in M}|z|_{\theta}$.

По условию точка $-\theta$ имеет единственную ближайшую в $(X,\|\cdot\|)$ точку $\widehat{y}$ из $M$. По доказанному выше $\|\widehat{y}+\theta\|=1$. Теперь, так как $B_{\theta}(0,1)=B(-\theta, 1)$, то $\widehat{y}$ - единственная ближайшая точка в норме $|\cdot|_{\theta}$ для 0 , т.е. $M$ - чебышёвское множество в пространстве $(X,|\cdot| \theta)$.

Пусть теперь $M$ - солнще в $(X,\|\cdot\|)$. Рассмотрим точку $x \notin M$. Вьше мы установили, что $\rho_{\theta}(x, M)>0$. Не ограничивая общности считаем $x=0$ и $\rho_{\theta}(0, M)=1$. Как и раньше, имеем $B_{\theta}(0,1)=B(-\theta, 1), \stackrel{\circ}{B}(-\theta, 1) \cap M=\varnothing, S(-\theta, 1) \cap M \neq \varnothing$. Пусть $\widehat{y}-$ солнечная точка в $(X,\|\cdot\|)$ для $-\theta$. По лемме А опорный конус

$$
\stackrel{\circ}{K}(\widehat{y},-\theta)=\bigcup_{\alpha>1} \stackrel{\circ}{B}(-\alpha \theta+(1-\alpha) \widehat{y}, \alpha)
$$


не пересекается с $M$. По предложению А имеем

$$
B(-\alpha \theta+(1-\alpha) \widehat{y}, \alpha)=\mathrm{H}_{\widehat{y}}^{\alpha}(B(-\theta, 1))=\mathrm{H}_{\widehat{y}}^{\alpha}\left(B_{\theta}(0,1)\right)=B_{\theta}(\widehat{y}(1-\alpha), \alpha)
$$

Поэтому опорньй конус

$$
\stackrel{\circ}{K}_{\theta}(\widehat{y}, 0)=\bigcup_{\alpha>1} \stackrel{\circ}{B}(\widehat{y}(1-\alpha), \alpha)
$$

не пересекается с $M$, а это по лемме А влечет, что $M$ - солнце в пространстве $\left(X,|\cdot|{ }_{\theta}\right)$.

Таким же образом мы устанавливаем утверждение леммы для строгих солнц.

2) Пусть $H$ - аффинное подпространство в $X$. Для простоты считаем $0 \in H$. Пусть $M \subset H$ - чебьшёвское множество в $(X,\|\cdot\|)$. Установим, что $M$ сохраняет это свойство в пространстве $\left(H,|\cdot|_{H, \theta}\right)$.

Пусть $x \in H \backslash M$. Зафиксируем $\theta \in \stackrel{\circ}{B}$. Поскольку $M-$ чебьшёвское множество в $(X,\|\cdot\|)$, то $\rho_{H, \theta}(0, M)>0$. Для простоты считаем $\rho_{H, \theta}(0, M)=1$.

Имеем $\stackrel{\circ}{B}_{H, \theta}(0,1) \cap M=\varnothing$. По определению $\stackrel{\circ}{B}_{H, \theta}(0,1)=\stackrel{B}{B}(-\theta, 1) \cap H$. Поскольку $M \subset H$, то $\stackrel{\circ}{B}(-\theta, 1) \cap M=\varnothing$. Далее, из того, что $\rho_{H, \theta}(0, M)=1$, имеем $S(-\theta, 1) \cap M \neq \varnothing$, и, так как $M$ - чебышёвское множество в $(X,\|\cdot\|)$, это пересечение состоит из одной точки. Поэтому $M$ - чебьшёвское множество в $\left(H,\left.|\cdot|\right|_{H, \theta}\right)$.

Теперь пусть $M \subset H$ - солнще в $(X,\|\cdot\|)$. Как и вьше, считаем $0 \in H \backslash M$, $\rho_{H, \theta}(0, M)=1$. Пусть $\widehat{y}-$ солнечная точка в $(X,\|\cdot\|)$ из $M$ для 0 . Понятно, что $0 \in S_{H, \theta}(0,1)$ и $0 \in S(-\theta, 1)$. Из леммы А имеем, что $K(\widehat{y},-\theta) \cap M=\varnothing$. Применяя предложение $\mathrm{A}$, из этого получаем, что $\stackrel{\circ}{K}_{H, \theta}(\widehat{y}, 0) \cap M=\varnothing$, а это в силу леммы А влечет, что $M$ - солнце в пространстве $\left(H,|\cdot|_{H, \theta}\right)$. Для строгих солнц рассуждения аналогичны. Лемма 1 доказана.

Напомним, что подмножество $M$ пространства $X$ называется ацикличным, если оно обладает тривиальной группой чеховских когомологий с коэффициентами из нетривиальной групшы ${ }^{1}$. Множество $M$ назьвается $P$-ацикличным, если для любой точки $x$ множество $P_{M} x$ ее ближайших точек из $M$ ациклично. Отметим, что замкнутое множество $M$ в конечномерном пространстве является $P$-ацикличньм, если и только если пересечение $M \cap B(x, r)$ ациклично для любых $x \in X, r>0$ (см. [6, с. 110]).

Из леммы 1 и из известного факта, что солнце в двумерном пространстве $X$ является $P$-ацикличным множеством (см., например, $[16],[17$, гл. 2], [7]), мы получаем следующее утверждение в симметричном случае и при условии $\operatorname{dim} X<\infty$ (доказанное Брауном в $[7, \S 4])$.

СЛЕДСТВИЕ 1. Пусть $X$ - линейное несимметрично нормированное пространство, $H-$ подпространство в $X, \operatorname{dim} H=2$, и пусть $M \subset H-$ солние в $X$. Тогда множество $M$ Р-ациклично.

Отметим, что ограниченно компактное (в частности, замкнутое множество в конечномерном пространстве) $P$-ацикличное множество в банаховом пространстве является солнцем $[1$, теорема 4.4]. Однако вопрос, будет ли произвольное солнце в произвольном конечномерном линейном нормированном пространстве $X P$-ацикличным (или хотя бы

\footnotetext{
${ }^{1} \mathrm{~K}$ примеру, стягиваемое (в частности, выпуклое) компактное подмножество линейного нормированного пространства ациклично; ацикличное множество связно; гомеоморфный образ ацикличного множества ацикличен.
} 
$P$-связным) до сих пор открыт [3], [7] (ответ на этот вопрос известен лишь, если $X-$ гладкое пространство (тогда каждое солнще в $X$ вьпукло), и в случаях $X=\ell^{\infty}(n)$, $X=c_{0}$ или при $\left.\operatorname{dim} X \leqslant 2\right)$.

4. Выпуклость чебышёвских множеств, лежащих в подпространстве $H$. В этом разделе для исследования выпуклости чебышёвского множества $M \subset H$, $\operatorname{dim} H<\infty$, в пространстве $X$ мы используем два подхода. Каждьй из них опирается на лемму 1. При первом подходе, рассматривая семейство сечений $(\stackrel{\circ}{B}-\theta) \cap H, \theta \in \stackrel{\circ}{B}$, шара $B$ подпространством $H$, нам достаточно найти хотя бы одно "хорошее" сечение $B{ }_{H, \widehat{\theta}}$ такое, что в пространстве $\left(H,|\cdot|_{H, \widehat{\theta}}\right)$ все чебьшёвские множества выпуклы. Поскольку по лемме 1 множество $M$ является чебьшёвским в $\left(H,|\cdot|_{H, \widehat{\theta}}\right)$, то $M$ будет вьпукло. $\mathrm{K}$ примеру, это условие будет вьполнено, если сечение $B_{H, \widehat{\theta}}$ гладкое в $H$. Второй подходинтегрированньй. Мы его умеем применять лишь при $\operatorname{dim} H=2$. При таком подходевсе сечения $B_{H, \theta}$ могут быть “плохими" - в каждом из пространств $\left(H,|\cdot|_{H, \theta}\right)$ сушествует невыпуклое чебьшёвское множество, - однако любое чебьшёвское множество $M \subset H$ в $X$ оказьвается вьпуклым.

Рассмотрим первьй подход. Пусть $M \subset H$ - чебьшёвское множество в $X, \theta \in \stackrel{\circ}{B}$. Рассечем шар $B-\theta$ подпространством $H$. Получится шар $B_{H, \theta}$ в подпространстве $H$, он определяет несимметричную норму $|\cdot|_{H, \theta}$ на $H$. По лемме $1 M$ - чебьшёвское множество в $\left(H,|\cdot|_{H, \theta}\right)$. Теперь для решения вопроса о вьпуклости множества $M$ можно воспользоваться любыми достаточными условиями вьпуклости в $H$, которые имеются в нашем арсенале.

Tеорема 1. Пусть $H \subset X-$ подпространство, $\operatorname{dim} H<\infty$, и пусть $M \subset H-$ чебышёвское множество в $X$. Предположим, что найдется такая точка $\theta \in \stackrel{\circ}{B}$, что сечение $B_{H, \theta}=(B-\theta) \cap H$ удовлетворяет хотя бы одному из условии:

a) $\operatorname{sm} B_{H, \theta}=B_{H, \theta}$ (т.е. любая точка сферы $S_{H, \theta}$ является ее точкой гладкости);

б) если $\operatorname{dim} H=3$ или 4, то $\exp B_{H, \theta} \subset \operatorname{sm} B_{H, \theta}$ (m.е. любая достижимая точка шара $B_{H, \theta}$ является его точкой гладкости).

Тогда мнохество $M$ выпукло.

Аналогичный результат можно сформулировать для солнц $M$ в пространстве $X$, лежащих в некотором подпространстве $H \subset X$.

Солние $M$ выпукло, если шар $B$ можнно так рассечь подпространством $H$, что сечение $B_{H, \theta}$ будет гладким множеством в $H\left(\operatorname{sm} B_{H, \theta}=S_{H, \theta}\right)$.

Утверждение а) теоремы 1 следует из того, что в конечномерном пространстве $H$ с гладкой единичной сферой всякое чебышёвское множество вьпукло, а утверждение б) вытекает из следующей теоремы $\mathrm{A}$ - известного критерия вьпуклости чебьшёвского множества в пространстве размерности 3 или 4, которая была доказана в трехмерном случае Бердьшевым [18] для нормированных пространств $X$ и Брондстедом [8] для нормированных и несимметрично нормированных пространств, а в четырехмерном - Брауном [19] в симметричном случае и автором [20] - в несимметричном случае.

Tеорема А. Пусть $\operatorname{dim} X=3$ или 4. Тогда каждое чебышёвское множество в $X$ выпукло, если и только если $\exp B \subset \operatorname{sm} B$ (т.е. если каждая достижимая точка шара $B$ является точкой гладкости). 
Прежде, чем перейти к рассмотрению второго подхода, посмотрим, когда наша задача вырождена, т.е. когда в $H$ содержатся только одноточечные чебышёвские множества $M$ в $X$. Например, задача тривиальна, если $X=\ell^{\infty}(n)$, а $H$ - подпространство в $X$, параллельное некоторой собственной грани $F$ единичного куба $B, \operatorname{dim} H=\operatorname{dim} F$. Понятно, что в этом случае $M \subset H$ не может содержать более двух точек. Итак, зафиксировав $H, \operatorname{dim} H<\infty$, обозначим

$$
\operatorname{tr}_{H}(S)=\{A \subset S \mid A=S \cap(H+\theta) \text { для некоторого } \theta \in S\},
$$

- "след" сдвигов подпространства $H$ на единичной сфере $S$ в $(X,\|\cdot\|)$. K примеру, если $H$ - гиперплоскость, а $h \in X^{*}$ и $\operatorname{Ker} h=H$, то $\operatorname{tr}_{H}(S)$ состоит из двух достижимых граней сферы $S^{*}$, на которых $h$ достигает максимума и минимума на $S$.

Итак, предположим, что

$$
\operatorname{card} A=1 \quad \text { для всякого } A \in \operatorname{tr}_{H}(S) .
$$

Это условие гарантирует, что в $H$ всегда содержится нетривиальное чебьшёвское множество в $X$. Действительно, рассмотрим произвольное строго вьпуклое замкнутоеподмножество $M_{1} \subset H$. У тверждение, что $M_{1}$ - чебьшёвскоемножество в $(H,|\cdot|)$, где $|\cdot|-$ произвольная несимметричная норма на $H$, является классическим. Из этого и из леммы 1 следует, что если $x \notin M_{1}$ и $\stackrel{\circ}{B}(x, r) \cap H \neq \varnothing$, то $x$ имеет единственную ближайшую точку в $M_{1}$, а для остальных точек $x \notin M_{1}$ предыдущее утверждение выполнено в силу (6). Обратно, если в $H$ содержатся нетривиальные чебышёвские множества $M$ в $X$, то, понятно, $\max \left\{\operatorname{dim} A \mid A \in \operatorname{tr}_{H}(S)\right\}<\operatorname{dim} H$. Видно, что это условие и (6) не сходятся, однако здесь мы не ставим задачи характеризации подпространств $H$, которые содержат нетривиальное чебьшёвское множество $M$ в $X$.

Теперь напомним следующее обобщение задачи о выпуклости чебьшёвских множеств с классического случая одного пространства на случай системы пространств, рассмотренное нами в $[17$, гл. 2$]$.

Рассмотрим систему $\mathfrak{X}=\left\{\left(Y,\|\cdot\|_{i}\right)\right\}_{i \in I}$, где $Y$ - действительное линейноепространство, $\operatorname{dim} Y<\infty,\|\cdot\|_{i}$ - несимметричная норма на $Y(i \in I), I$ - некоторое множество индексов. Множество $M \subset Y$ называется чебышёвским относительно $\mathfrak{X}$, если $M$ является чебьшёвским множеством в пространстве $\left(Y,\|\cdot\|_{i}\right)$ для любого $i \in I$. Если $\mathfrak{X}$ состоит только из одного пространства, то это определение совпадает с обычным определением чебышёвского множества.

Пусть $S_{i}=S_{i}(0,1)$ - единичная сфера $i$-го пространства $\left(Y,\|\cdot\|_{i}\right), i \in I$. Система точек $\mathscr{P}:=\left\{p_{i} \in S_{i}\right\}_{i \in I}$ назьвается достижимой относительно $\mathfrak{X}$, если для тела $W_{\mathfrak{X}}:=\overline{\operatorname{conv}}\left(\bigcup_{i \in I}\left\{S_{i}-p_{i}\right\}\right)$ точка 0 принадлежит границе тела $W_{\mathfrak{X}}$ и является достижимой; система точек $\mathscr{P}$ назьвается гладкой, если точка $0 \in \mathrm{bd} W_{\mathfrak{X}}$ является точкой гладкости тела $W_{\mathfrak{X}}$.

В [17, теорема 2.1] мы охарактеризовали систему $\mathfrak{X}$ двумерных несимметрично нормированных пространств, в которой всякое чебышёвское множество относительно $\mathfrak{X}$ выпукло.

Теорема В. Пусть $\mathfrak{X}=\left\{\left(Y,\|\cdot\|_{i}\right)\right\}_{i \in I}$ - система двумерных несимметрично нормированных пространств. Для того, чтобы каждое чебышёвское относительно Х множество было выпукло, необходимо и достаточно, чтобы каждая достижимая относительно $\mathfrak{X}$ система точек $\left\{p_{i} \in S_{i}\right\}_{i \in I}$ была гладкой. 
Оказалось, что понятие множества, чебьшёвского относительно системы пространств, естественным образом возникает в задаче приближения множествами $M$, содержашимися в подпространстве $H$. Именно, сечения сдвигов $B-\theta$ единичного шара $B$ подпространством $H$ дают нам нужное семейство $\mathfrak{X}=\left\{\left(H,\|\cdot\|_{H, \theta}\right)\right\}_{\theta \in B}$. В силу леммы 1 множество $M$ чебышёвское относительно $\mathfrak{X}$.

Теорема 2. Пусть $H \subset X-$ подпространство, $\operatorname{dim} H=2$. Предположсим, что выполнено условие (6). Тогда следующие два условия әквивалентны:

а) любое чебишёвское мнохество $M \subset H$ в $X$ выпукло

б) любая достижимая относительно набора $\mathfrak{X}=\left\{\left(H,\|\cdot\|_{H, \theta}\right)\right\}_{\theta \in \dot{B}}$ система

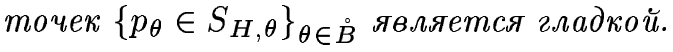

ДоказАтельство. Рассуждаем от противного. Установим а) $\Longrightarrow$ б). Предположим, что каждое чебьшёвское множество $M \subset H$ в $X$ выпукло и нашлась достижимая негладкая система точек $\mathscr{P}:=\left\{p_{\theta} \in S_{H, \theta}(0,1)\right\}_{\theta \in B^{\circ}}$. Имеем $0 \in \exp W_{\mathfrak{X}}, 0 \notin \operatorname{sm} W_{\mathfrak{X}}$, поэтому найдутся две различные опорные прямые $G_{1}$ и $G_{2}$ к телу $W_{\mathfrak{X}}$ в точке 0 такие, что $G_{i} \cap W_{\mathfrak{X}}=\{0\}, i=1,2$. Для $i \in\{1,2\}$ через $G_{i}^{+}$обозначим замкнутое полупространство с границей $G_{i}$, не содержащее $W_{\mathfrak{X}}$. Пусть $M:=G_{1}^{+} \cup G_{2}^{+}$. По лемме А множество $M$ - солнце, а так как $G_{i} \cap W_{\mathfrak{X}}=\{0\}$, то $M$ - чебышёвское множество в любом пространстве $\left(X,|\cdot|_{H, \theta}\right) \in \mathfrak{X}$. Из этого и из (6) вытекает, что $M$ - чебышёвское множество в $(X,\|\cdot\|)$. Мы получили противоречие с исходной посылкой, поскольку $M$ невыпукло.

Обратная импликация доказьвается с использованием леммы 1 и полностью аналогично рассуждениям, при доказательстве теоремы В [17, с. 56-58]. Теорема 2 доказана.

5. Выпуклость ограниченных чебышёвских множеств $M$, лежащих в гиперп лоскости $H$. Не так давно выяснилось, что проблема вьпуклости произвольных и проблема выпуклости произвольных ограниченных чебышёвских множеств оказываются различными - удивительный факт, предугаданньй С.Б. Стечкиньгм и установленный Царьковым (см. [4]-[6]). В [5] им, в частности, установлено, что в конечномерном $^{3}$ линейном нормированном пространстве всякое ограниченное чебьшёвское множество (ограниченное $P$-ацикличное множество) выпукло тогда и только тогда, когда $\overline{\exp } S^{*}=S^{*}$.

Рассмотрим аналогичньй вопрос о выпуклости ограниченных чебьшёвских множеств $M$ в $X$ при условии $M \subset H, H$ - гиперплоскость в $X$. Ниже, как обычно, $\operatorname{cl} A$ - замькание множества $A$.

Teорема 3. Пусть $\operatorname{dim} X<\infty, h \in X^{*}, H=\operatorname{Ker} h \subset X-$ zиперплоскость $u$ пусть $M \subset H$-ограниченное чебышёвское мнохсество в $X$. Тогда если найдется $\theta \in \stackrel{\circ}{B}$ такое, что выполнено

$$
\operatorname{cl}\left\{\varphi \in S_{H, \theta}^{*} \mid \exists f \in \exp S^{*} u \exists \lambda=\lambda(f)>0 \text { такие, ито }\left.f\right|_{H}=\lambda \cdot \varphi\right\}=S_{H, \theta}^{*},
$$

где $S_{H, \theta}^{*}-$ единичная сфера пространства, сопряженного $\kappa\left(H,|\cdot|_{H, \theta}\right)$, то множество $M$ выпукло.

\footnotetext{
${ }^{2}$ Напомним, что по (6) в $H$ всегда существуют неодноточечные чебышёвские множества в $X$.

${ }^{3}$ В бесконечномерном случае этот вопрос исследован в [4].
} 
СЛЕДСтвиЕ 2. Пусть $\operatorname{dim} X<\infty, H-$ zиперплоскость в $X$ u nусть $M \subset H-$ ограниченное чебышёвское мнохсество в $X$. Если хотя бы для одного из функиионалов $h_{1}, h_{2} \in S^{*}$, определяюших $H$, выполнено $h_{i} \in \operatorname{int}_{S^{*}} \overline{\exp }\left(S^{*}\right), i=1$ или 2 , то множество $M$ выпукло.

Прокомментируем эти утверждения. Результат Царькова, в частности, утверждает, что если “практически каждая" опорная гиперплоскость к сфере $S$ содержит в пересечении с $S$ точку гладкости сферы $S$, то любое ограниченное чебьшёвское множество $M$ в $X$ вьпукло. Сходным образом теорема 3 показьвает, что каждое ограниченное чебьшёвское множество $M \subset H$ в $X$ выпукло, если для некоторого $\theta \in \stackrel{\circ}{B}$ для “практически каждой" гиперплоскости Г в $H$, опорной к $S_{H, \theta}$, найдется гиперплоскость $G$ в $X$, опорная к $S$, “параллельная" Г и пересекающаяся с $S$ по гладкой точке. Мы докажем теорему 3 чуть ниже.

Следующая лемма достаточно интуитивно ясна: полукруг не вьпуклый; следовательно, существуют гиперплоскости, отсекающие кусочки от концов полукруга; полусфера также не вьпукла, и поэтому возможно отсечь гиперплоскостью от нее обод [6, c. 107]. Эти две ситуации являются типичными.

Лемма В (И.Г. Царьков [5], [6, с. 107]). Пусть $M \subset X, \operatorname{dim} X<\infty$. Если множество $M$ ограничено, замкнуто, невыпукло и $X \backslash M$ связно, то существует открытое полупространство $\stackrel{\circ}{\mathrm{G}} \subset X$ и мнохество $\Sigma \subset \stackrel{\circ}{\mathrm{G}} \cap \mathrm{M}$ такие, что

(i) $\Sigma$ является относительной границей некоторого выпуклого мнохества $u$, следовательно, $\Sigma$ гомеоморфно конечномерной сфере; $u$

(ii) $\Sigma$ является ретрактом $\stackrel{\mathrm{G}}{\mathrm{n}} \mathrm{M}$.

\section{Также нам понадобится}

ЛЕмма С (см., например, [6, с. 107]). Пусть $M \subset X-$ чебышёвское множсество с непрерывной метрической проекиией $u x \in X, r>0$. Предположим, что $B(x, r) \cap M \neq \varnothing$. Тогда $B(x, r) \cap M$ есть ретракт шара $B(x, r)$.

ДоКАЗАТЕЛЬСТВо ТЕОРЕМЫ 3. ПредПоложим, что множество $M$ невыпукло. По лемме $1 M$ является чебышёвским множеством в $\left(H,|\cdot|_{H, \theta}\right)$. Из того, что в конечномерном пространстве $X$ чебьшёвское множество является солнщем немедленно следует, что дополнение к чебышёвскому множеству в $H$ связно, и поэтому, используя лемму $\mathrm{C}, \mathrm{\kappa} M$ можно применить лемму В. Выберем $\stackrel{\circ}{G}$-полупространство в $H$ и множество $\Sigma \subset \stackrel{G}{\cap} \cap M$, как в лемме В. Без ограничения общности считаем $0 \in \operatorname{bd} \stackrel{G}{G}$. Выберем $\theta \in \stackrel{\circ}{B}$ таким образом, чтобы выполнялось (7).

Пусть $g \in S_{H, \theta}^{*}-$ такой функционал, что $\operatorname{Ker} g=\operatorname{bd} \stackrel{\circ}{G}$ и $g(\Sigma)<0$. По условию (7) для любого $\varepsilon>0$ можно выбрать такой функционал $f \in \exp S^{*}$, что $\Sigma \subset\{z \mid f(z)<0\}$ и $\left.(\lambda f)\right|_{H} \in \mathscr{O}_{\varepsilon}(g)$ (т.е. $\left.|\lambda f-g|_{H, \theta}<\varepsilon\right)$, где константа $\lambda=\lambda(f)$ выбрана из $(7)$.

Обозначим $F=\operatorname{Ker} f, \stackrel{\circ}{F}=\{z \in X \mid f(z)<0\}$. Теперь из (7) следует, что если гиперплоскость $F$ является опорной к некоторому шару $B(y, r)$ и $B(y, r) \subset \stackrel{\circ}{F}$, то пересечение $F \cap B(y, r)$ обязательно содержит некоторую точку гладкости $v$ шара $B(y, r)$. Далее, поскольку $v \in \operatorname{sm} B(y, r)$, то определенньй в (5) опорный конус $\stackrel{\circ}{K}(v, y)$ к шару $B(y, r)$ является открытьм полупространством, очевидно, совпадающим с $\stackrel{F}{F}$. Поскольку в конечномерном пространстве чебышёвское множество является солнцем, то лемма А нам дает, что $\stackrel{\circ}{K}(v, y) \cap M=\varnothing$. Выбирая $\alpha$ в (5) достаточно большим и обозначая $b=\stackrel{\circ}{B}(-\alpha v+(\alpha+1) y,(\alpha+1)\|y-v\|)$, имеем $\Sigma \subset(b \cap M) \subset(\stackrel{\circ}{F} \cap M)$. 
Итак, поскольку $\Sigma \subset b \cap M$, то по (ii) $\Sigma$ - ретракт $b \cap M$, а по лемме С множество $M \cap b$ - ретракт шара $b$. Мы получили противоречие, поскольку по (i) $\Sigma$ гомеоморфно конечномерной сфере, а такая сфера не может быть ретрактом шара $b$. Теорема 3 доказана.

Утверждение теоремы 4 берет начало от результата Царькова [5], построившего норму $\|\cdot\|_{T}$ на линейном пространстве $X, 3 \leqslant \operatorname{dim} X<\infty$, относительно которой всякое ограниченное чебьшёвское множество в $X$ вьпукло, в то время как существует невыпуклое неограниченное чебьшёвское множество в $X$.

Tеорема 4. Пусть $\operatorname{dim} X<\infty, H \subset X-$ гиперплоскость в $X, \operatorname{dim} H \geqslant 3$. Тогда на $X$ существует норма, относительно которой любое ограниченное чебышёвское мнохество $M \subset H$ в $X$ будет выпукло, однако найдется неограниченное невыпуклое чебышёвское мнохсество $M_{1} \subset H$ в $X$.

ДоказАтЕльство. Рассмотрим на $H$ норму $\|\cdot\|_{T}$, построенную Царьковым в [5, c. 85] (см. также [7]). Им показано, что любое ограниченное чебьшёвское множество в $\left(H,\|\cdot\|_{T}\right)$ выпукло и там же построен пример невыпуклого неограниченного чебышёвского множества $M_{1}$ в $\left(H,\|\cdot\|_{T}\right)$. Используя этот пример, распространим норму $\|\cdot\|_{T}$ с $H$ на $X$, оставив множество $M_{1} \subset H$ без изменения.

Рассмотрим на $X$, отождествив его с $H \oplus \mathbb{R}$, норму $\|\cdot\|_{C}$, определяемую функционалом Минковского следуюшего тела $C$ (бипирамиды):

$$
C=\operatorname{conv}\left\{(0,1),(0,-1),\left(B_{T}, 0\right)\right\}, \quad \text { где } B_{T}-\text { единичный шар в } H \text { в норме }\|\cdot\|_{T} \cdot
$$

В $[21$, замечание 8.4] показано, что любое сечение тела $C$ аффинной гиперплоскостью $H+(0, \alpha),|\alpha| \leqslant 1$, есть сдвиг гомотетии $\mathrm{H}_{0}^{1-|\alpha|}\left(B_{T}\right)$ шара $B_{T}$ с коэффициентом $1-|\alpha|$ и центром в 0 :

$$
C \cap(H+\alpha)=\mathrm{H}_{0}^{1-|\alpha|}\left(B_{T}\right)+(0, \alpha)=(1-|\alpha|) B_{T}+(0, \alpha), \quad|\alpha| \leqslant 1 .
$$

Пусть теперь $M \subset H$ - произвольное чебьшёвское множество в $\left(H,\|\cdot\|_{T}\right)$. Рассмотрим произвольную точку $x \in X \backslash M$. Пусть $r=\rho_{C}(x, M):=\inf _{z \in M}\|z-x\|_{C}(r>0$, поскольку $M$ замкнуто). Имеем два случая.

Пусть сначала $\stackrel{\circ}{B}_{C}(x, r) \cap H \neq \varnothing$. По (8) сечение $B_{C}(x, r) \cap H$ гомотетично шару $B_{T}$; поэтому, так как $M$ - чебышёвское множество в $\left(H,\|\cdot\|_{T}\right)$, точка $x$ имеет единственную ближайшую из $M$ в норме $\|\cdot\|_{C}$. Пусть теперь $\stackrel{\circ}{B}_{C}(x, r) \cap H=\varnothing$. Поскольку $\operatorname{dim} H<\infty$, то $S_{C}(x, r) \cap H \neq \varnothing$, и в силу определения бипирамиды $C$ это пересечение одноточечно и, понятно, принадлежит $M$. В этом случае $x$ также имеет единственную ближайшую точку из $M$.

Итак, $M_{1}$ лежит в $H$ и является невьпуклым неограниченным чебышёвским множеством в $\left(X,\|\cdot\|_{C}\right)$. С другой стороны, из цитированного вьше утверждения Царькова для $\left(H,\|\cdot\|_{T}\right)$ следует, что любое ограниченное чебышёвское множество в $\left(X,\|\cdot\|_{C}\right)$, которое лежит в $H$, выпукло. Отметим, что в $H$ содержатся нетривиальные ограниченные чебьшёвские множества $M$ в $X$ (вьпуклые по доказанному). В качестве примера $M$ можно взять произвольное строго вьпуклое ограниченное подмножество в $H$. Теорема 4 доказана. 


\section{СПИСОК ЦИТИРОВАННОЙ ЛИТЕРАТУРЫ}

[1] Власов Л. П. Аппроксимативные свойства множеств в линейных нормированных пространствах // УМН. 1973. Т. 28. №6. С. 3-66.

[2] Балаганский В.С., Власов Л.П. Проблема выпуклости чебышёвских множеств // УМН. 1996. Т. 51. №6. С. 125-188.

[3] Карлов М.И., Царьков И. Г. Выпуклость и связность чебышёвских множеств и солнц // Фундамент. и прикл. матем. 1997. Т. 3. №4. С. 967-978.

[4] Царьков И. Г. Компактные и слабо компактные чебышёвские множества в линейных нормированных пространствах // Тр. МИАН. 1989. Т. 189. С. 169-184.

[5] Царьков И.Г. Ограниченные чебьшёвские множества в конечномерных банаховых пространствах // Матем. заметки. 1984. Т. 36. №1. С. 73-86.

[6] Brown A. L. Chebyshev sets and the shapes of convex bodies // Methods of Functional Analysis in Approximation Theory / ed. C. A. Micchelli. Internat. Series of Numerical Math. V. 76. Proc. Intern. Conf. (Indian Inst. Techn. Bombay, 16-20.XII.1985). Basel: Birkhäuser, 1986. P. 97-121.

[7] Brown A. L. On the problem of characterising suns in finite-dimensional spaces // Rend. Circ. Mat. Palermo (2) Suppl. 2002. V. 68. № 1. P. 315-328.

[8] Brøndsted A. Convex sets and Chebyshev sets, II // Math. Scand. 1966. V. 17. № 1. P. 5-15.

[9] Бородин П. А. Теорема Банаха-Мазура для пространств с несимметричной нормой и ее приложения в выпуклом анализе // Матем. заметки. 2001. Т. 69. № 3. С. 329-337.

[10] Ефимов Н. В., Стечкин С. Б. Некоторые свойства чебышёвских множеств // Докл. АН CCCP. 1958. T. 118. №1. C. 17-19.

[11] Ошман Е. В. Чебышёвские множества и непрерывность метрической проекции // Изв. вузов. Матем. 1970. №9. С. 78-82.

[12] Козко А. И. Многомерные неравенства разных метрик в пространствах с несимметричной нормой // Матем. сб. 1998. Т. 189. № 9. С. 85-106.

[13] Künzi H.-P. A. Nonsymmetric distances and their associated topologies: About the origin of basic ideas in the area of asymmetric topology // Handbook of the History of General Topology / ed. C.E. Aull, R. Lowen. V. 3. Dordrecht: Kluwer, 2001. P. 853-968.

[14] Магарил-Ильяев Г. Г., Тихомиров В. М. Выпуклый анализ и его приложения. М.: Эдиториал УССР, 2000.

[15] García-Raffi L. M., Romaguera S., Sánchez-Pérez E. A. Sequence spaces and asymmetric norms in the theory of computational complexity // Math. Comput. 2002. V. 36. P. 1-11.

[16] Berens H., Hetzelt L. Suns and contractive retracts in the plane // Теория приближений функций. Труды межд. конф. (Киев, 31 мая -5 июня, 1983) / ред. Н. П. Корнейчук и др.. М.: Наука, 1987. C. 483-487.

[17] Алимов А. Р. Аппроксимативные свойства множеств в линейных пространствах с несимметричной нормой. Дисс. . . . к. ф.-м. н. М.: МГУ, 1997.

[18] Бердышев В.И.К вопросу о чебышёвских множествах // Докл. АН АзССР. 1966. Т. 22. № 9. C. 3-5.

[19] Brown A. L. Chebyshev sets and facial systems of convex sets in finite-dimensional spaces // Proc. London Math. Soc. 1980. V. 41. P. 297-339.

[20] Алимов А.Р. О структуре дополнения к чебышёвским множествам // Функцион. анализ и прилож. 2001. Т. 35. № 3. С. 19-27.

[21] Лейхтвейс К. Вьпуклые множества. М.: Наука, 1985.

Московский государственный университет им. М.В. Ломоносова

Поступило

E-mail: alimov@shade.msu.ru

03.02 .2004

Исправленный вариант

22.11 .2004 